\title{
Numerical Simulation of Flow Field of DN2400 Hedge Dissipator
}

\author{
Tan Huipan ${ }^{1}$,Sun Jianghe ${ }^{2}$, Liao Zhifang ${ }^{3}$,Zhang Baiyun ${ }^{1}$,Tang Zherun ${ }^{1}$, Jiang Jin ${ }^{1}$, a \\ ${ }^{1}$ Key Lab of Hydraulic Machinery Transient, MOE, Wuhan University, Wuhan, China \\ ${ }^{2}$ Gansu Province Water Conservancy and Hydropower Survey and Design Institute, Lanzhou, China \\ ${ }^{3}$ Bensv Valve Stock co., ltd, Tianjin, China
}

\begin{abstract}
The distribution of water resources in China is uneven. Long distance water conveyance projects have effectively solved this problem. In the water delivery system, the energy dissipator plays an important role in pressure regulation and regulation. Therefore, the design and structure of the energy dissipator affect the working efficiency and stable operation of the whole water delivery system, and it is of great significance to study the internal flow field of the energy dissipator. Based on a emergency project, this paper studies the internal flow field of the energy dissipation valve in A location and B location in the design conditions, accident conditions and water separation conditions to verify the DN2400 hedging energy dissipation. The rationality of the structural design of the device provides a reference for the design and improvement of the energy dissipator in the future.
\end{abstract}

\section{Introduction}

\subsection{Hedging energy absorber overview}

The valve is a general-purpose machine[1], which is widely used in the chemical, hydraulic and other industries, and is one of the indispensable equipment for the pipeline fluid delivery system[2]. The energy dissipation valve is one of the valves. This paper studies the hedged energy dissipation valve, which is mainly used to reduce pressure and regulate flow. Such a device is generally a combination of two different diameter pipes (spool sleeve and outer pipe), Wherein a plurality of vertical circular holes are arranged on the circumference of the small diameter inner tube, that is, the valve core sleeve, When the water enters the energy dissipation valve and fills the spool sleeve and the outer tube, the water flow flows into the valve core sleeve through the circular hole provided in the circumference of the valve sleeve, and then flows in the water supply direction.

\subsection{Research status of energy dissipation valve}

For the study of energy-dissipation valves, domestic experts and scholars have started early on the research of their working characteristics and energy dissipation principles, and have achieved some results. Xue Yongfei, Yang Jiwei and other experts and scholars have carried out theoretical analysis on the flow characteristics and resistance characteristics of the energy dissipation valve. The concept of the drag coefficient of the energy dissipation valve is proposed and the formula of flow calculation is derived. Based on the above theory, the key factors affecting the energy dissipation characteristics of the energy dissipation valve are analyzed, Based on this, an overall evaluation method for the energy dissipation characteristics of the energy dissipation valve is proposed[3-6]. Scholar Lian Zhanghua et al[7]. performed flow field analysis on two different types of high-pressure throttle valves, and found that the use time of the wedgeshaped throttle valve is longer than that of the tapered throttle valve, and after optimizing the structure of the wedge throttle valve, the optimal structure used by the wedge throttle valve is obtained; Yuan Xiuqian [8] and other scholars used the rigorous mathematical method to obtain a simple formula that can calculate the valve overflow according to the flow characteristics of the energydissipating valve and obtain the verification through experiments.

Foreign experts and scholars began to study the flow characteristics of the energy dissipation valve and the structural design and improvement of the energy dissipation valve earlier. Foreign research on energysaving valves started relatively early. As early as around 1960 , foreign scholars began to explore the flow and cavitation characteristics of valves[9-16]. Pountney D C [12] and other scholars used the k-e turbulence model to determine the flow characteristics of the orifice valve and proposed a comparable experimental study. Chern M J [14] and other scholars have prevented the generation of cavitation by changing the structure of the suction valve. At the same time, it is proposed that the cavitation type can be used for cavitation prediction of other control valves.

a Corresponding author: Jiang Jin, 00010482@whu.edu.cn 


\subsection{Project Overview}

\section{(1) Project A}

The over-flow of the design condition of hedge dissipator is $7.5 \mathrm{~m}^{3} / \mathrm{s}$, the accident condition is $10.5 \mathrm{~m}^{3} / \mathrm{s}$, and the flow over-flow condition is $2.5 \mathrm{~m}^{3} / \mathrm{s}$; The pressure in front of each valve is $8.95 \mathrm{~m}$, and the pressure behind each valve is $7.97 \mathrm{~m}$.

(2) Project B

The over-flow of the design condition of hedge dissipator is $7.5 \mathrm{~m}^{3} / \mathrm{s}$, the accident condition is $10.5 \mathrm{~m}^{3} / \mathrm{s}$, and the flow over-flow condition is $2.5 \mathrm{~m}^{3} / \mathrm{s}$; The pressure in front of each valve is $4.79 \mathrm{~m}$, and the pressure behind each valve is $3.34 \mathrm{~m}$.

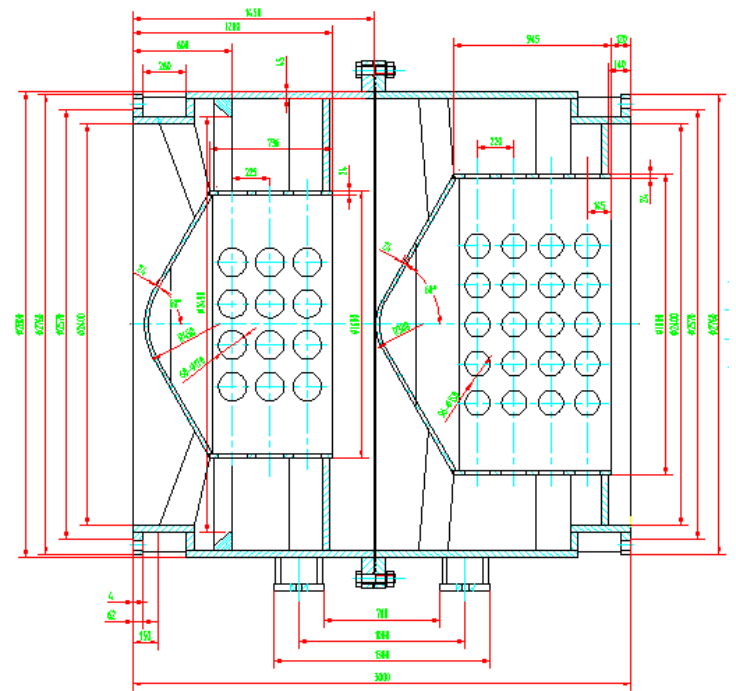

Fig.1 two dimensional structure diagram of energy dissipator

\subsection{Contents of research}

First,using Pro/Engineer software to build a threedimensional model of the energy dissipation valve based on two-dimensional drawings,; Then use ICEM software and Pumplinx software to divide the grid; finally, ICEM software and Pumplinx software are used to solve the calculation. The flow field simulated by two different softwares is compared and analyzed to verify the rationality of the structure design of the energy dissipation valve, which provides a reference for the structure design and improvement of the energy dissipation valve in the future.

\section{Geometric Modeling of DN2400 Hedge Absorber}

Using Pro/Engineer software, according to the twodimensional drawings of DN2400 hedge damper, the three-dimensional geometric model is established.

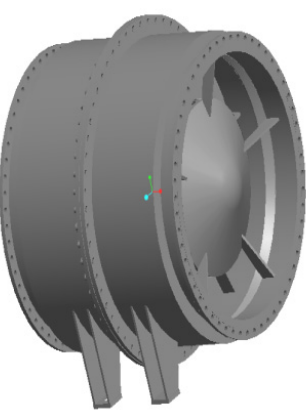

(a) the overall import model

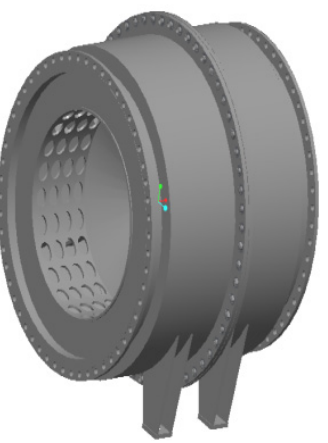

(b) the overall export model Fig.2 Three-dimensional structure diagram

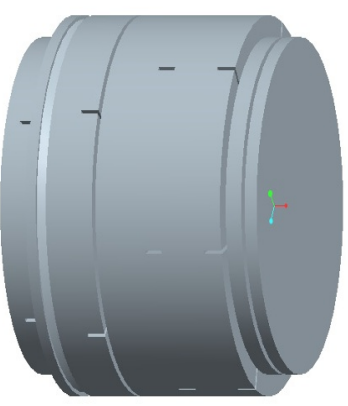

(a) overall

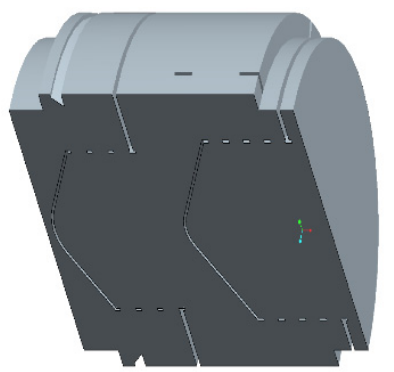

(b) cross-section
Fig.3 Three-dimensional watershed map

\section{DN2400 Hedge Dissipator Meshing}

According to the three-dimensional geometric model of the DN2400 hedging energy dissipator established above, the ICEM software and the Pumplinx software are used for meshing.

\subsection{Divide the grid with ICEM software}

Using ICEM software, the unstructured tetrahedral mesh with strong adaptability to the shape of the runner is used to divide the grid of the hedged energy absorber. After the meshing is completed, the mesh quality is checked for each part of the flowing water body, and finally the total number of meshes in the calculation area of the full flow field model is 3.574 million. The well-divided grid looks like this: 

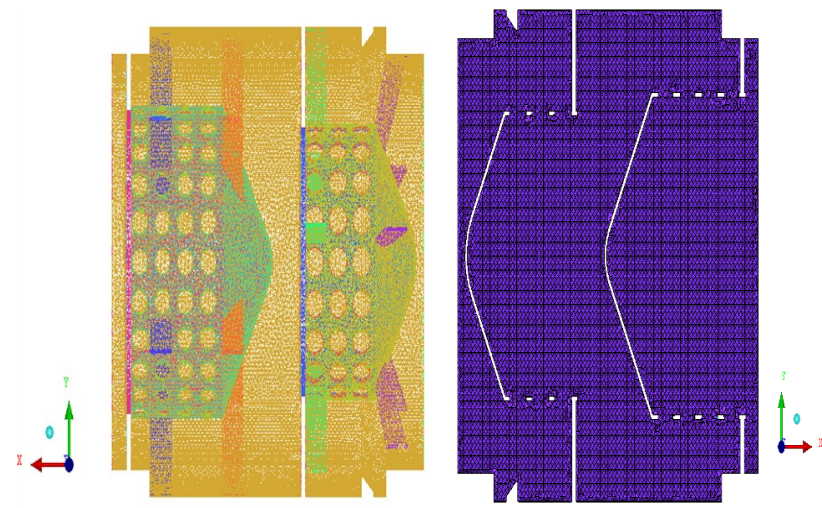

(a) overall mesh distribution of the model (b) profile mesh distribution

Fig.4 DN2400 Hedge Dissipator Meshing

\subsection{Divide the grid with Pumplinx software}

Pumplinx uses a Cartesian grid technique based on the binary tree method to mesh it and automatically encrypts it in areas with large curvature. After the meshing is completed, the mesh quality is checked for each part of the flowing water body, and finally the total number of meshes in the calculation area of the full flow field model is about 1.2 million. The well-divided grid looks like this:
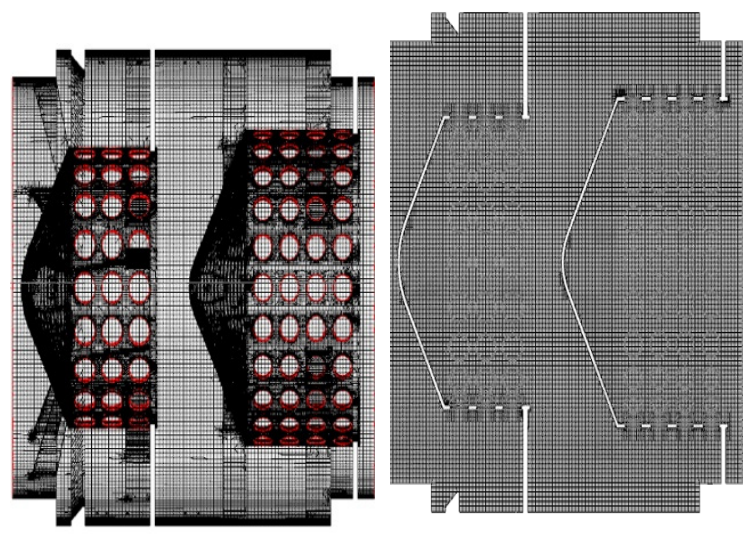

(a) overall mesh distribution of the model (b) profile mesh distribution Fig.5 DN2400 Hedge Dissipator Meshing

\section{CFD solver settings}

In order to ensure the accuracy of the numerical simulation, the DN2400 hedging energy dissipator will be numerically simulated by Fluent software and Pumplinx software, and the results will be compared.

\subsection{Turbulence model}

The numerical simulation of the DN2400 hedging energy eliminator uses the RNG k- $\varepsilon$ model. The RNG k- $\varepsilon$ model is derived from rigorous statistical techniques. These small-scale motion systems are systematically removed from the governing equation by embodying small-scale effects in large-scale motion and modified viscosity terms. The equations are expressed as follows:

$$
\begin{aligned}
& \frac{\partial(\rho k)}{\partial t}+\frac{\partial\left(\rho k u_{i}\right)}{\partial x_{i}}=\frac{\partial}{\partial x_{j}}\left[\alpha_{k} \mu_{e f f} \frac{\partial k}{\partial x_{j}}\right]+G_{k}+\rho \varepsilon \\
& \frac{\partial(\rho \varepsilon)}{\partial t}+\frac{\partial\left(\rho \varepsilon u_{i}\right)}{\partial x_{i}}=\frac{\partial}{\partial x_{j}}\left[\alpha_{\varepsilon} \mu_{e f f} \frac{\partial \varepsilon}{\partial x_{j}}\right]+\frac{C_{1 \varepsilon}^{*} \varepsilon}{k} G_{k}-C_{2 \varepsilon} \rho \frac{\varepsilon^{2}}{k}
\end{aligned}
$$

In the formula, $\mu$ eff is the effective turbulent viscosity and is the sum of $\mu$ and $\mu \mathrm{t}$. The main features of the RNG $\mathrm{k}-\varepsilon$ model are:

(1) By correcting the turbulent viscosity, the rotation and swirl flow in the average flow are considered.

(2) Adding an item to the $\varepsilon$ equation reflects the mainstream time-average strain rate and effectively improves the accuracy.

(3) The RNG theory provides an analytical formula for the turbulent Prandtl number, while the commonly used standard $\mathrm{k}-\varepsilon$ model is the user-provided constant.

(4) Equivalent to the standard Re-number model of the standard k- $\varepsilon$ model, RNG theory provides an analytical formula that considers the flow viscosity of low Re numbers. The role of these formulas depends on the correct treatment of the near-wall area. ( Simulated by wall function method or $\mathrm{k}-\varepsilon$ model with low Re number)

It can be seen that the RNG $\mathrm{k}-\varepsilon$ model can better handle the flow with high strain rate and large streamline bending, including complex shear flow with fast strain, medium vortex and partial transition (Such as boundary layer separation, block separation, vortex rear step 
separation, etc) . These characteristics make the RNG k$\varepsilon$ model have higher reliability and accuracy in flow field simulation. Therefore, in order to make the calculation of the flow field of the DN2400 hedging dissipator more accurate, we use the RNG k- $\varepsilon$ turbulence model for this calculation.

\subsection{Boundary conditions}

For the numerical simulation of the DN2400 hedging energy eliminator, setting the correct boundary conditions is an important guarantee for calculation accuracy.

\subsection{1 boundary conditions in fluent}

(1) import boundary conditions: pressure import

Regardless of the compressibility of the water, when using the Fluent software for calculation, the simulated inlet boundary conditions of the inlet and the total pressure of the energy absorber are set according to different working conditions..

(2) Export boundary conditions: pressure outlet

Determine the outlet pressure value of the absorber under different operating conditions as shown in the table below as the boundary condition of the outlet.

\begin{tabular}{|c|c|c|c|}
\hline $\begin{array}{c}\text { Working } \\
\text { condition }\end{array}$ & $\begin{array}{c}\text { Imported 1. Project A } \\
\text { water head } \\
(\mathbf{m})\end{array}$ & $\begin{array}{c}\text { Export } \\
\text { water } \\
\text { head } \\
\mathbf{( m )}\end{array}$ & $\begin{array}{c}\text { flow } \\
(\mathbf{m} \mathbf{3} / \mathbf{s})\end{array}$ \\
\hline $\begin{array}{c}\text { Design } \\
\text { condition }\end{array}$ & 8.95 & 7.97 & 7.5 \\
\hline $\begin{array}{c}\text { Accident } \\
\text { condition }\end{array}$ & 8.95 & 7.97 & 10.5 \\
\hline $\begin{array}{c}\text { Water } \\
\text { separation } \\
\text { condition }\end{array}$ & 8.95 & 7.97 & 2.5 \\
\hline
\end{tabular}

Table2. Project B

\begin{tabular}{|c|c|c|c|}
\hline $\begin{array}{c}\text { Working } \\
\text { condition }\end{array}$ & $\begin{array}{c}\text { Imported } \\
\text { water head } \\
(\mathbf{m})\end{array}$ & $\begin{array}{c}\text { Export } \\
\text { water } \\
\text { head } \\
\mathbf{( m )}\end{array}$ & $\begin{array}{c}\text { flow } \\
\left(\mathbf{m}^{3} / \mathbf{s}\right)\end{array}$ \\
\hline $\begin{array}{c}\text { Design } \\
\text { condition }\end{array}$ & 4.79 & 3.34 & 7.5 \\
\hline $\begin{array}{c}\text { Accident } \\
\text { condition }\end{array}$ & 4.79 & 3.34 & 10.5 \\
\hline $\begin{array}{c}\text { Water } \\
\text { separation } \\
\text { condition }\end{array}$ & 4.79 & 3.34 & 2.5 \\
\hline
\end{tabular}

\subsubsection{Pumplinx boundary conditions}

(1) Import boundary conditions: pressure import

Regardless of the compressibility of the water, when using the Pumplinx software for calculations, the simulated inlet boundary conditions are set according to the different inlet pressures of the energy dissipator.

(2) Export boundary conditions: flow exit

The flow rate of $2.5 \mathrm{~m}^{3} / \mathrm{s}$ in the water separation condition is set as the flow boundary condition.

\section{Numerical simulation results of DN2400 hedging energy absorber}

\subsection{Simulation of the DN2400 hedging energy eliminator using Fluent software}

\subsubsection{Project $A$}

Flow field analysis of the outlet of the energy dissipator :

(1) Simulation results of the energy dissipator in the design condition (inlet head is $8.95 \mathrm{~m}$, outlet head is $7.97 \mathrm{~m}$, flow rate is $7.5 \mathrm{~m}^{3} / \mathrm{s}$.
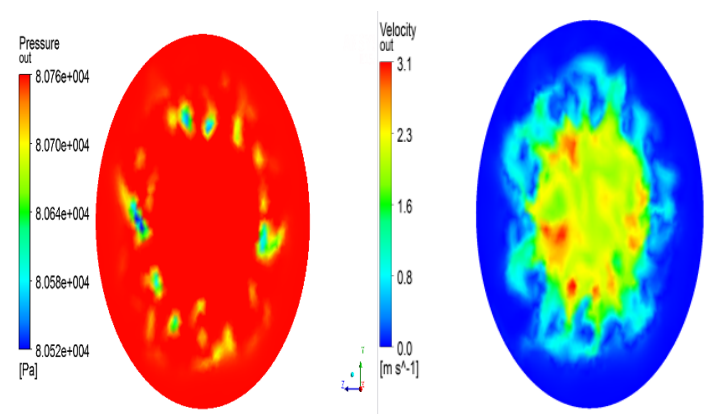

(a) Export pressure cloud map (b) Export flow rate cloud map

(2) Simulation results of the energy dissipator in the accident condition (inlet head is $8.95 \mathrm{~m}$, outlet head is $7.97 \mathrm{~m}$, flow rate is $10.5 \mathrm{~m}^{3} / \mathrm{s}$ )
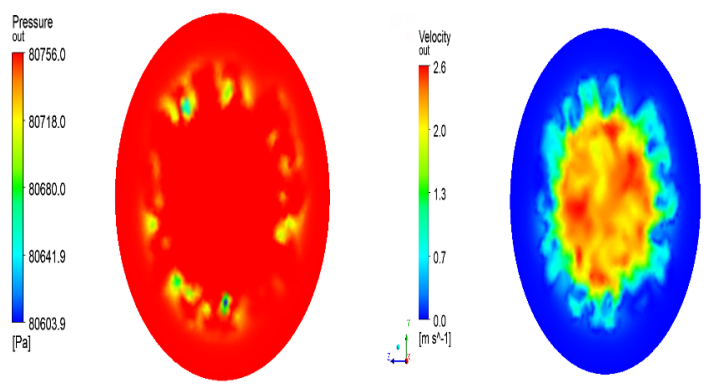

(a) Export pressure cloud map (b) Export flow rate cloud map

(3) Simulation results of the energy dissipator in the water separation condition (inlet head is $8.95 \mathrm{~m}$, outlet head is $7.97 \mathrm{~m}$, flow rate is $2.5 \mathrm{~m}^{3} / \mathrm{s}$ )
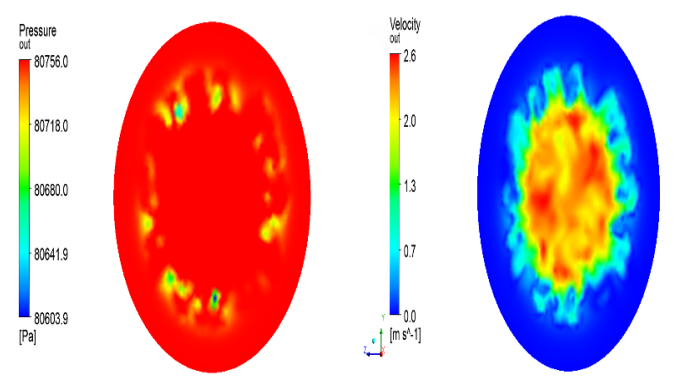

(a) Export pressure cloud map (b) Export flow rate cloud map

Fig.6 Energy Dissipator Exit Cloud

From the cloud diagram of the outlet of the energy dissipator in Figure 5.1, it can be seen that the outlet 
pressure of each working condition is relatively stable and the pressure value is similar. The outlet flow velocity distribution shows a similar flow velocity at the center of the tube and gradually decreases toward the periphery. The flow velocity near the tube wall is close to zero.

Flow field analysis of $\mathrm{z}=0$ cross-section energy dissipator:

Design condition

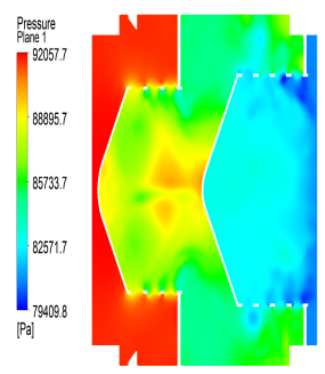

(a) Pressure cloud map

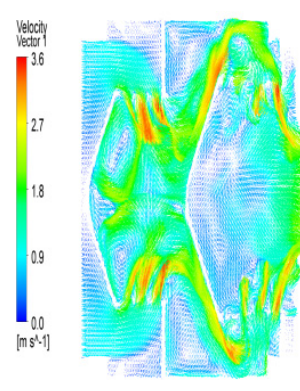

(c) velocity vector

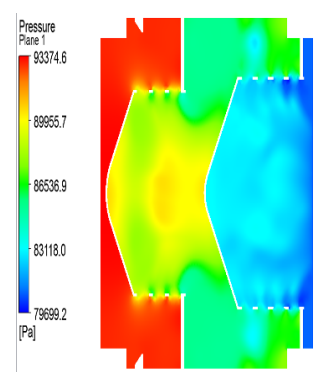

(a) Pressure cloud map

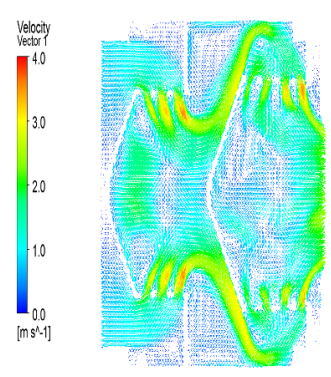

(c) velocity vector Accident condition
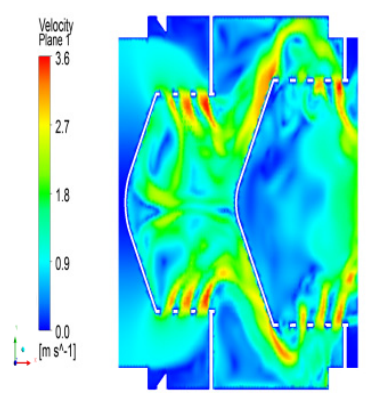

(b) Speed cloud map
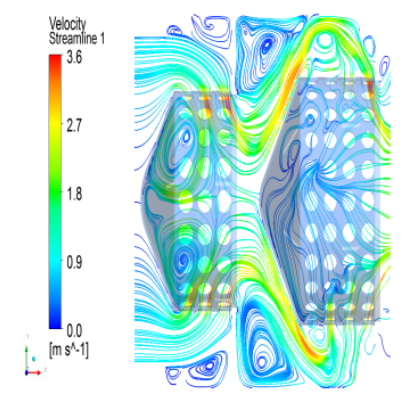

(d) streamline diagram
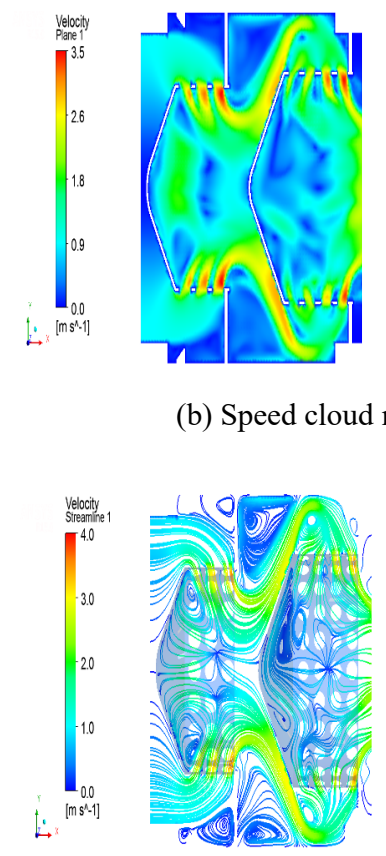

(d) streamline diagram
Water separation condition (b) Speed cloud map
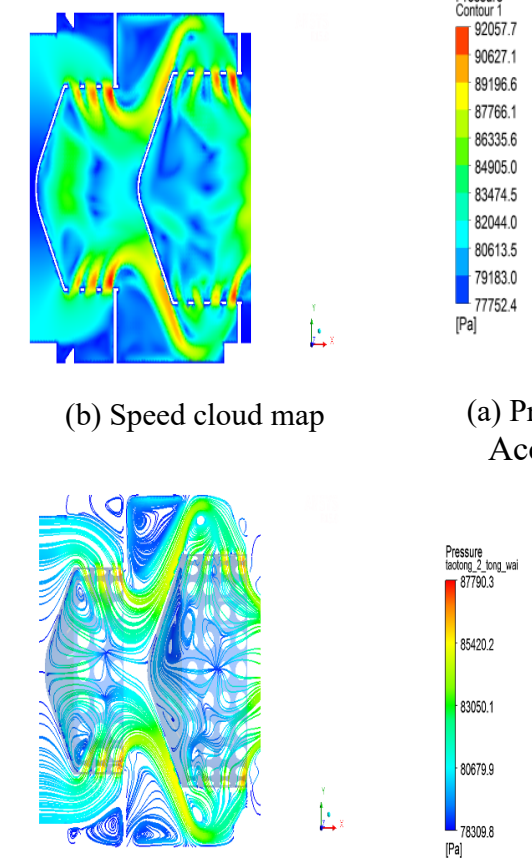

Water separation condition

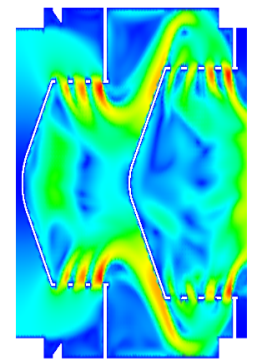

(b) Speed cloud map

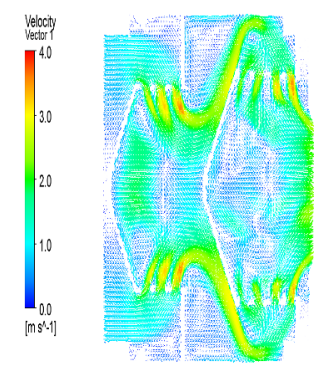

(c) velocity vector
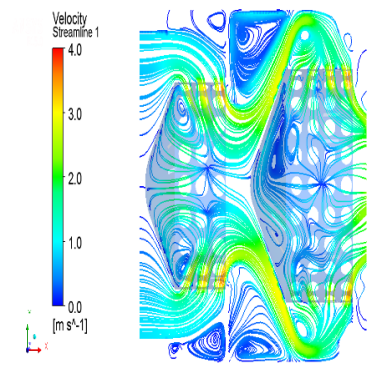

(d) streamline diagram
It can be seen from the graph of $5.2 \mathrm{z}=0$ cross-section that the pressure of each working condition is reduced by the energy dissipator. The center pressure of the tube between the primary and secondary sleeves is higher than the surrounding; when the water flows through the sleeve hole The speed is large; it can be seen in the streamline diagram that vortices appear before and after the baffle.

Energy dissipator sleeve pressure cloud diagram and streamline:

Design condition

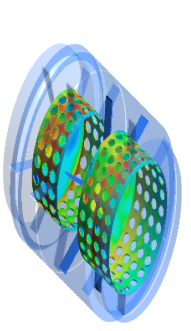

(a) Pressure cloud map

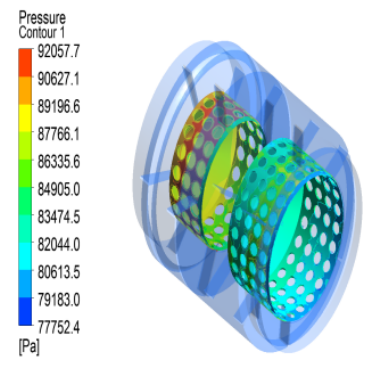

(a) Pressure cloud map Accident condition

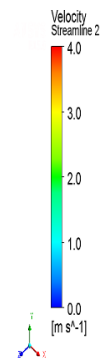

(b) Streamline diagram

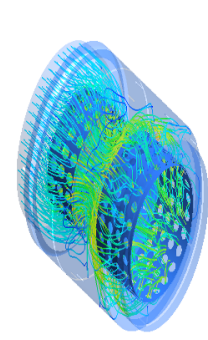

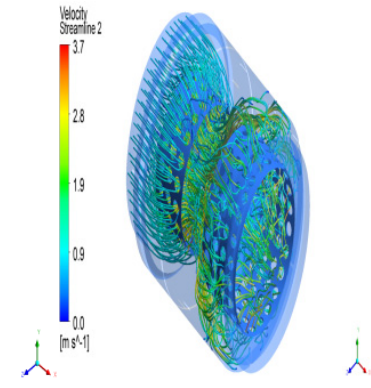

(b) Streamline diagram
. 

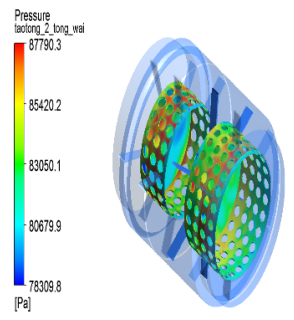

(a) Pressure cloud map
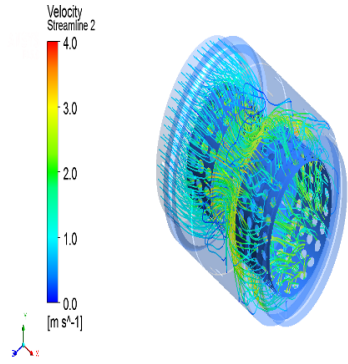

(b) Streamline diagram
Pressure Cloud and Streamline

From the pressure cloud diagram and the flow line of the energy dissipator sleeve in Figure 5.3, it can be seen that the pressure distribution of the energy absorber sleeve is relatively uniform under each working condition; the flow diagram shows that there is a vortex at the sleeve baffle, and the movement is more complicated.

\subsubsection{Project $A$}

Flow field analysis of the outlet of the energy dissipator : (1) Simulation results of the energy dissipator in the design condition (inlet head is $8.95 \mathrm{~m}$, outlet head is $7.97 \mathrm{~m}$, flow rate is $7.5 \mathrm{~m}^{3} / \mathrm{s}$.
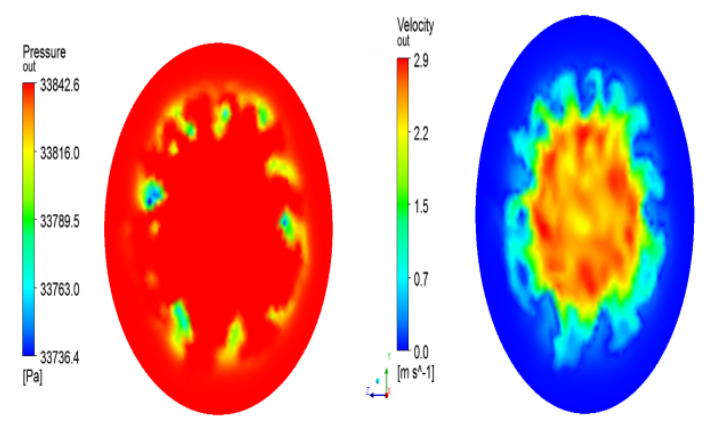

(a) Export pressure cloud map

(b) Export flow rate cloud map

(2) Simulation results of the energy dissipator in the accident condition (inlet head is $8.95 \mathrm{~m}$, outlet head is $7.97 \mathrm{~m}$, flow rate is $10.5 \mathrm{~m}^{3} / \mathrm{s}$ )
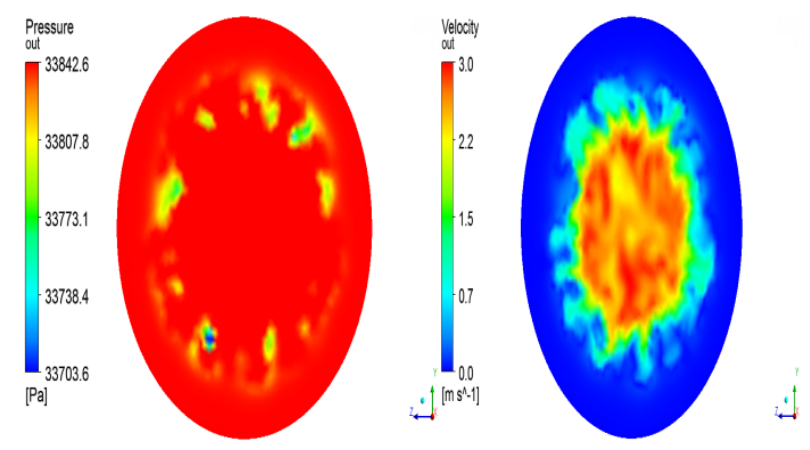

(a) Export pressure cloud map (b) Export flow rate cloud map (3) Simulation results of the energy dissipator in the water separation condition (inlet head is $8.95 \mathrm{~m}$, outlet head is $7.97 \mathrm{~m}$, flow rate is $2.5 \mathrm{~m}^{3} / \mathrm{s}$ )
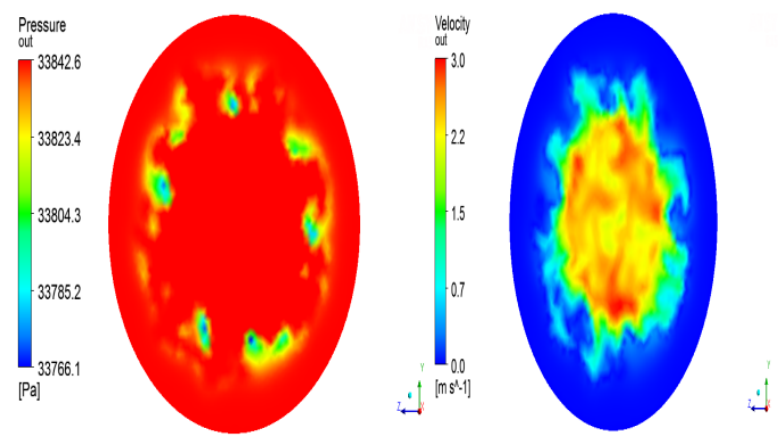

(a) Export pressure cloud map (b) Export flow rate cloud map Fig.9 Energy Dissipator Exit Cloud

It can be seen from the cloud diagram of the energy dissipator in Figure 5.4 that the outlet pressures of each working condition are relatively stable and the pressure values are similar. The outlet flow velocity distribution is similar to that of the tube center flow velocity, which gradually decreases toward the periphery, and the flow velocity near the pipe wall is close to zero.

Flow field analysis of $\mathrm{z}=0$ cross-section energy dissipator:

Design condition
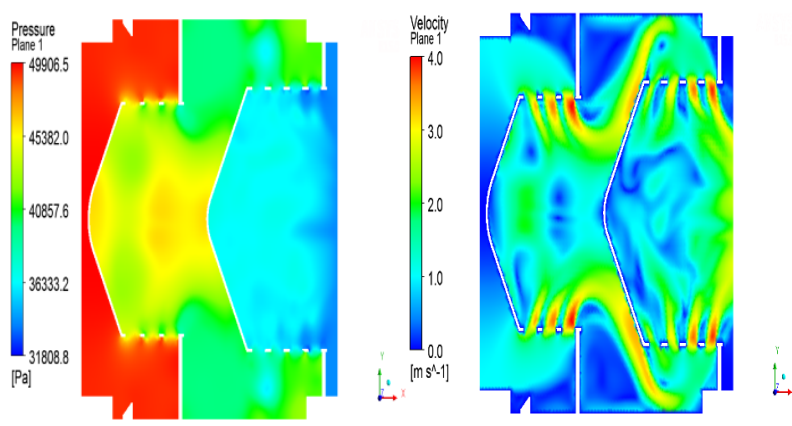

(a) Pressure cloud map

(b) Speed cloud map
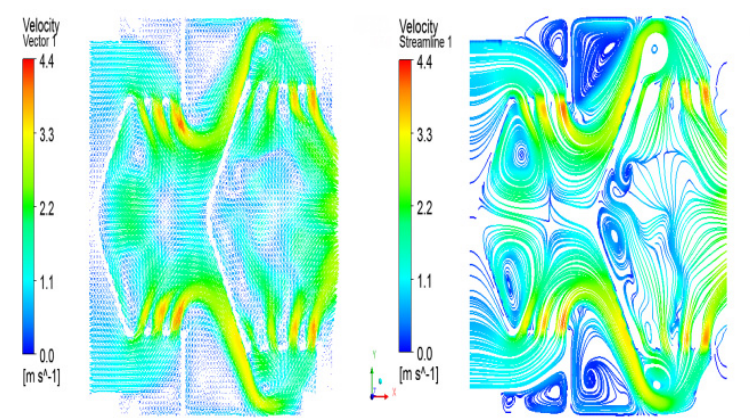

(c) velocity vector Accident condition (d) streamline diagram 


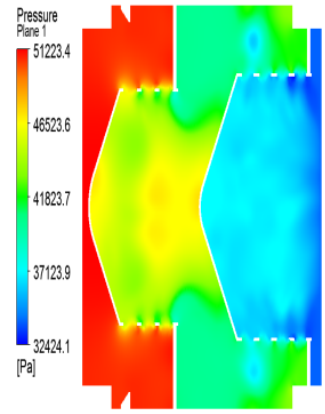

(a) Pressure cloud map

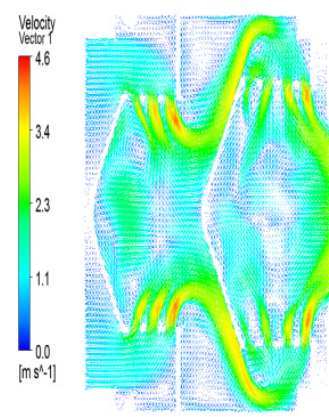

(c) velocity vector

Water separation condition

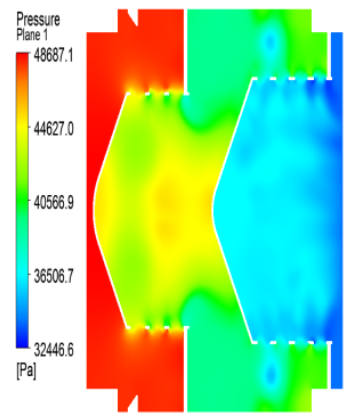

(a) Pressure cloud map
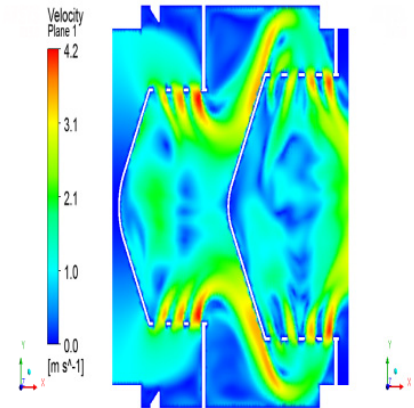

(b) Speed cloud map
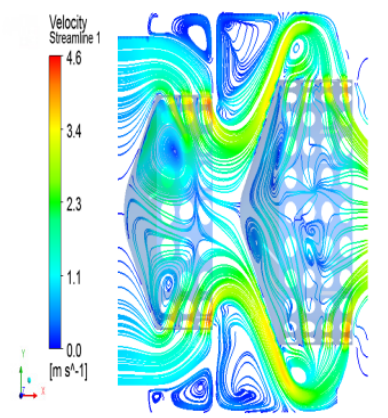

(d) streamline diagram
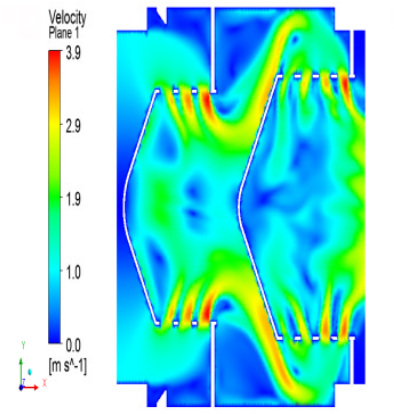

(b) Speed cloud map

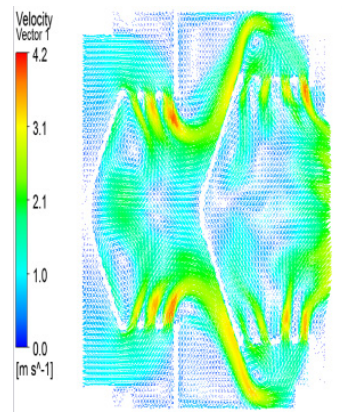

(c) velocity vector
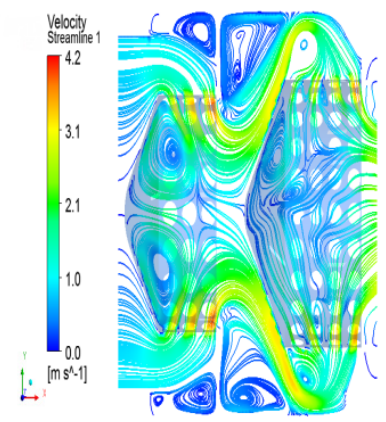

(d) streamline diagram

Fig. $10 \mathrm{z}=0$ cross-section cloud

It can be seen from the graph of $\mathrm{z}=0$ cross-section cloud in Figure 5.5 that the pressure of each working condition is reduced by the energy dissipator. The center pressure of the tube between the primary and secondary sleeves is higher than the surrounding; when the water flows through the sleeve hole, the speed is large; in the streamline diagram, it can be seen that vortex appears before and after the baffle.

Energy dissipator sleeve pressure cloud diagram and streamline:

Design condition
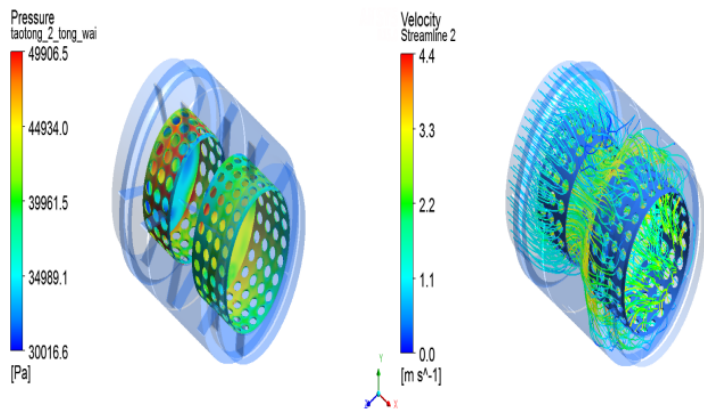

(a) Pressure cloud map

(b) Streamline diagram

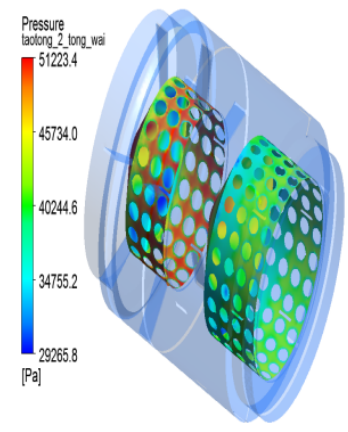

(a) Pressure cloud map

Water separation condition

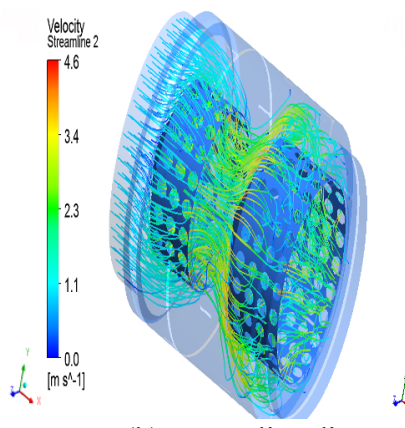

(b) Streamline diagram

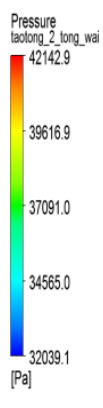

(a) Pressure cloud map

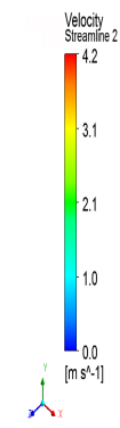

(b) Streamline diagram

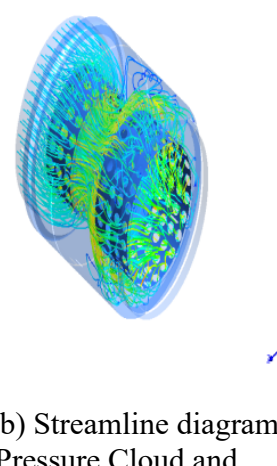

\section{Streamline}

From the pressure cloud diagram and the flow line of the energy dissipator sleeve in Figure 5.6, it can be seen that the pressure distribution of the energy absorber sleeve is relatively uniform under each working condition; the flow diagram shows that there is a vortex at the sleeve baffle, and the movement is more complicated.

\subsubsection{Summary of simulation results}


(1) Project A

Table3 Results of simulating the energy absorber using Fluent

\begin{tabular}{|c|c|c|}
\hline \multicolumn{3}{|c|}{ software } \\
Condition & $\begin{array}{c}\text { flow } \\
\left(\mathbf{m}^{3} / \mathbf{s}\right)\end{array}$ & $\begin{array}{c}\text { Computational } \\
\text { flow }\left(\mathbf{m}^{\mathbf{3}} / \mathbf{s}\right)\end{array}$ \\
\hline $\begin{array}{c}\text { Design } \\
\text { condition }\end{array}$ & 7.5 & 2.77 \\
\hline $\begin{array}{c}\text { Accident } \\
\text { condition }\end{array}$ & 10.5 & 2.87 \\
\hline $\begin{array}{c}\text { Water } \\
\text { separation } \\
\text { condition }\end{array}$ & 2.5 & 2.57 \\
\hline
\end{tabular}

(2)

Table4 Results of simulating the energy absorber using Fluent

\begin{tabular}{|c|c|c|}
\hline Condition & $\begin{array}{l}\text { flow } \\
\left(\mathbf{m}^{3} / \mathbf{s}\right)\end{array}$ & $\begin{array}{l}\text { Computational } \\
\text { flow }\left(\mathrm{m}^{3} / \mathbf{s}\right)\end{array}$ \\
\hline $\begin{array}{c}\text { Design } \\
\text { condition }\end{array}$ & 7.5 & 3.24 \\
\hline $\begin{array}{l}\text { Accident } \\
\text { condition }\end{array}$ & 10.5 & 3.38 \\
\hline $\begin{array}{c}\text { Water } \\
\text { separation } \\
\text { condition }\end{array}$ & 2.5 & 3.11 \\
\hline
\end{tabular}

\subsection{Simulation results of DN2400 hedging energy dissipator using Pumplinx software}

\subsubsection{Project $A$}

After the inlet pressure $8.95 \mathrm{~m}$ and outlet flow $2.5 \mathrm{~m}^{3} / \mathrm{s}$, the results are as follows:

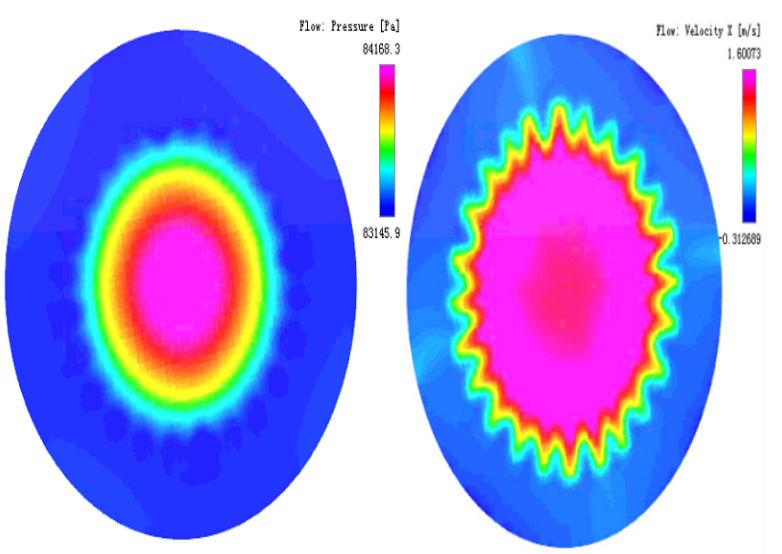

(a) outlet pressure cloud map

(b) exit velocity cloud map Fig.12 outlet of energy dissipator

From the cloud diagram of the energy dissipator in Fig. 12 , it can be seen that the outlet pressure is large in the middle and small in the periphery due to the different velocity, and the outlet velocity is large in the middle and gradually decreases on both sides.

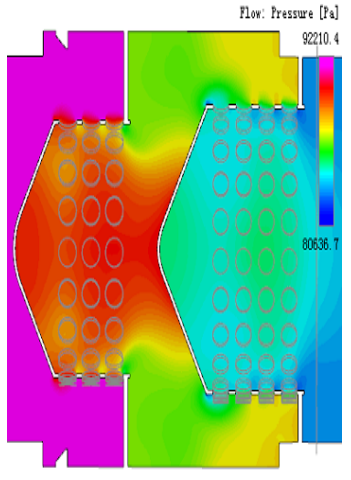

(a) pressure cloud chart

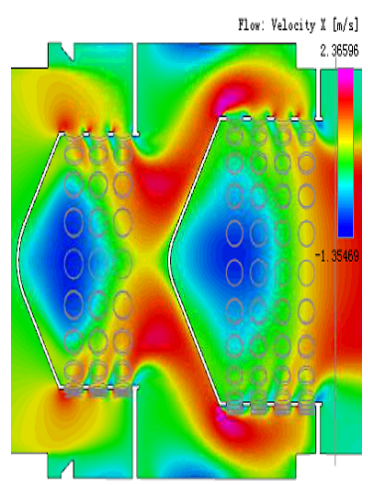

(b) velocity cloud chart
Fig.13 cross section cloud chart of $y=0$

From the cross-sectional cloud chart of Fig. $5.8 \mathrm{y}=0$, it can be seen that the pressure decreases from the inlet through the energy dissipator as a whole, and increases slightly before the secondary sleeve due to the resistance effect; when the flow velocity passes through the sleeve hole, the velocity is larger, and there is reflux phenomenon in the sleeve.

\subsubsection{Project $B$}

After the inlet pressure $4.79 \mathrm{~m}$ and outlet flow $2.5 \mathrm{~m}^{3} / \mathrm{s}$, the results are as follows:
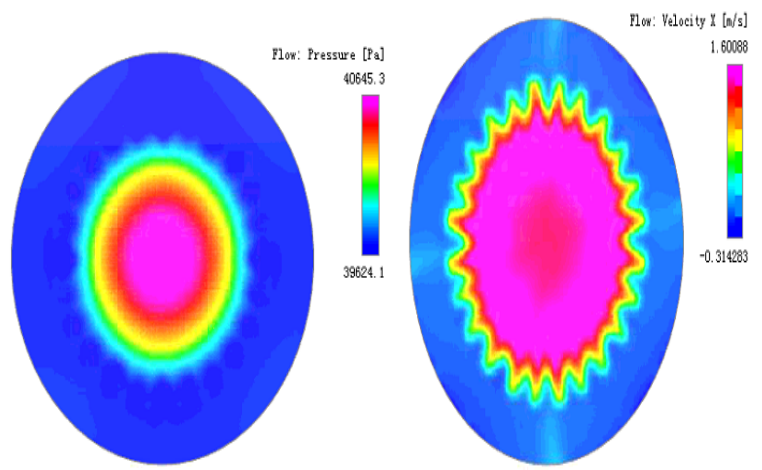

(a) outlet pressure cloud map

(b) exit velocity cloud map Fig.14 outlet of energy dissipator

From the cross-section energy dissipator diagram of Fig. $5.9 \mathrm{y}=0$, it can be seen that the outlet pressure, due to the difference of velocity, presents the middle large peripheral small pressure, the outlet velocity presents the middle large velocity, the two sides gradually reduce the trend. 


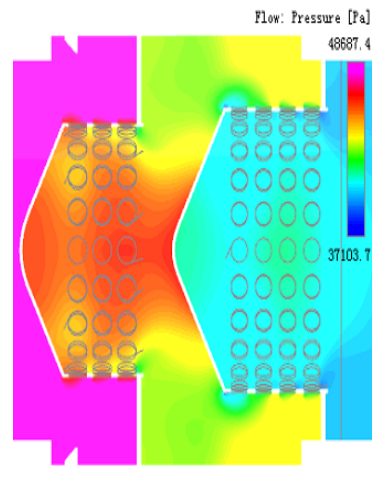

(a) pressure cloud chart

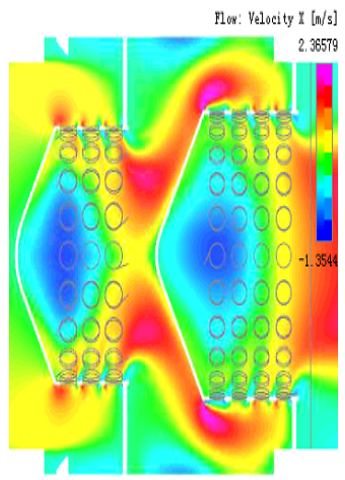

(b) velocity cloud chart
From the cross-section cloud chart of Fig. $5.10 \mathrm{y}=0$, it can be seen that the pressure decreases from the inlet through the energy dissipator as a whole, and increases slightly before the secondary sleeve due to the resistance effect, and the velocity is higher when the sleeve hole flows.

\subsubsection{Summary of simulation results}

The simulation results of the energy dissipator under different working conditions are summarized in the following table.

(1) Project A

Table5 Results of simulating the energy absorber using Pumplinx software

\begin{tabular}{|c|c|c|c|}
\hline Condition & $\begin{array}{c}\text { Imported } \\
\text { water head } \\
\text { (m) }\end{array}$ & $\begin{array}{c}\text { Exported } \\
\text { water } \\
\text { head } \\
\text { (m) })\end{array}$ & $\begin{array}{c}\text { Computational outlet } \\
\text { pressure (m) }\end{array}$ \\
\hline $\begin{array}{c}\text { Water } \\
\text { separation } \\
\text { condition }\end{array}$ & 8.95 & 7.97 & \\
\hline
\end{tabular}

(2) Project B

Table6 Results of simulating the energy absorber using Pumplinx software

\begin{tabular}{|c|c|c|c|}
\hline & & & \\
Condition & $\begin{array}{c}\text { Imported } \\
\text { water head } \\
(\mathrm{m})\end{array}$ & $\begin{array}{c}\text { Exported } \\
\text { water } \\
\text { head } \\
(\mathrm{m})\end{array}$ & $\begin{array}{c}\text { Computational outlet } \\
\text { pressure (m) }\end{array}$ \\
& & (m) & \\
\hline
\end{tabular}

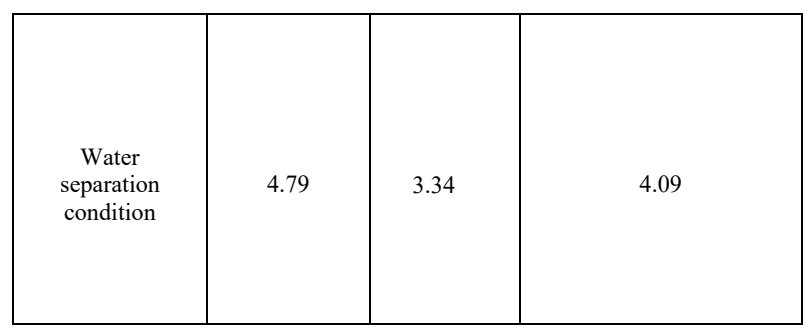

\section{SUMMARY}

The simulation results of the energy dissipator under different working conditions are summarized in the following table.

Table7 Results of simulating the energy absorber using Fluent

\begin{tabular}{|c|c|c|c|}
\hline & $\begin{array}{c}\text { Conditio } \\
\mathbf{n}\end{array}$ & $\begin{array}{c}\text { flow } \\
\left(\mathrm{m}^{3} / \mathrm{s}\right)\end{array}$ & $\begin{array}{l}\text { Computational } \\
\text { flow }\left(\mathrm{m}^{3} / \mathrm{s}\right)\end{array}$ \\
\hline \multirow{3}{*}{ Project A } & $\begin{array}{l}\text { Design } \\
\text { condition }\end{array}$ & 7.5 & 2.77 \\
\hline & $\begin{array}{l}\text { Accident } \\
\text { condition }\end{array}$ & 10.5 & 2.87 \\
\hline & $\begin{array}{c}\text { Water } \\
\text { separation } \\
\text { condition }\end{array}$ & 2.5 & 2.57 \\
\hline \multirow{3}{*}{ Project B } & $\begin{array}{l}\text { Design } \\
\text { condition }\end{array}$ & 7.5 & 3.24 \\
\hline & $\begin{array}{l}\text { Accident } \\
\text { condition }\end{array}$ & 10.5 & 3.38 \\
\hline & $\begin{array}{c}\text { Water } \\
\text { separation } \\
\text { condition }\end{array}$ & 2.5 & 3.11 \\
\hline
\end{tabular}

Table8 Results of simulating the energy absorber using

\begin{tabular}{|c|c|c|c|}
\hline & Pumplinx software \\
\hline Condition & $\begin{array}{c}\text { flow } \\
\left(\mathbf{~ m}^{\mathbf{3}} / \mathbf{s}\right)\end{array}$ & $\begin{array}{c}\text { Computational } \\
\text { outlet pressure } \\
(\mathbf{m})\end{array}$ \\
\hline Project A & $\begin{array}{c}\text { Water } \\
\text { separation } \\
\text { condition }\end{array}$ & 2.5 & 8.36 \\
\hline $\begin{array}{c}\text { Water } \\
\text { Project B } \\
\text { separation } \\
\text { condition }\end{array}$ & 2.5 & 4.09 \\
\hline
\end{tabular}

\section{References}

1. Ming Cidong. Application Technology of Regulating Valves[J]. Chemical Industry Automation and Instruments, 1993(1): 61-65. 
2. Lu Peiwen, Sun Xiaoxia, Yang Yuliang. Valve Selection Manual [M]. Mechanical Industry Press, 2016.

3. Xue Yongfei. Control Analysis of Flow Characteristics of Regulating Valves[J]. Journal of Henan Institute of Engineering(Natural Science), 2003, 15(2): 19-23.

4. Yang Jiwei. Evaluation of Flow Regulation Characteristics of Regulating Valves[J]. Valve, 1999(4): 11-15.

5. Yang Jiwei, Zhang Lirong. Analysis of Resistance Characteristics of Regulating Valves[J]. Valve, 2001(2): 22-24.

6. Yang Jiwei. Theoretical Study on Flow Regulation of Regulating Valves[J]. Fluid Machinery, 2003, 31(2): 24-26.

7. Lian Zhanghua, Liu Gan, Gong Jianwen, et al. Flow field analysis and structural improvement of high pressure throttle valve[J]. Petroleum Machinery, 2004, 32(9): 22-24.

8. Fu Weidong, Yuan Xiuqian, Huang Bencheng, et al. Calculation method of regulating valve flow $[\mathrm{J}]$. Valve, 1999(1): 4-6.

9. Corbett, Richard A. New control valve improves operating characteristics[J]. The Oil and Gas Journal. Dec 16, 1985, Vol. 83, p92-95.

10. Wang G, Liu S, Shintani M, et al. Study on Cavitation Damage Characteristics around a Hollow-Jet Valve.[J]. Jsme International Journal, 1999, 42(4):649-657.

11. ] Husu, Martti. Trimming control valve noisecavitation[J]. InTech. Dec 1988, Vol. 35 Issue 12, p38-39.

12. Pountney D C, Weston W, Banieghbal M R. A numerical study of turbulent flow characteristics of servo-valve orifices[J]. Archive Proceedings of the Institution of Mechanical Engineers Part A Journal of Power Engineering, 1989, 203(203):139-147.

13. Yu Z, Xiaorui G. Relationship Between the Forces on the Spool and Anti-cavitation Performance and Throttling Effect after the Notch of the Valve[J]. Journal of Applied Sciences, 2013, 13(10):16771683.

14. Chern M J, Hsu P H, Cheng Y J, et al. Numerical Study on Cavitation Occurrence in Globe Valve[J]. Journal of Energy Engineering, 2013, 139(1):25-34.

15. Wilson J, Boelen E L. PREVENTING CAVITATION REQUIRES PROPER DESIGN AND OPERATION OF FEED-WATER VALVES[J]. Power Engineering, 2003, 107(11).

16. Adolph T. High cavitation in minimal space[J]. 2005, 95(11):62-63. 